\title{
Healthy, natural, efficient and tunable lighting: four-package white LEDs for optimizing the circadian effect, color quality and vision performance
}

\author{
Ji Hye Oh, Su Ji Yang and Young Rag Do
}

To date, most current reports on the development and optimization of artificial lighting sources have focused on the energy performance levels and limited color qualities of white light-emitting diodes (LEDs). However, these properties are insufficient in terms of representing all performance levels required when adjusting white LEDs for healthy and smart lighting. Here, we introduce essential and advanced figures of merit pertaining to circadian performance as well as vision performance and color quality. We compare all possible properties of commercialized artificial lighting, daylight and four-package white LEDs which consist of long-wavelength pass dichroic filter (LPDF)-capped, phosphor-converted red, amber and green LEDs (pc-LEDs) and a blue LED. We show that these tunable four-package white LEDs produce a tunable circadian effect for melatonin suppression/secretion, a high color quality for color perception/reproduction, high efficiency for energy savings and tunable figures of merit for the smart LED lighting market.

Light: Science \& Applications (2014) 3, e141; doi:10.1038/lsa.2014.22; published online 14 February 2014

Keywords: circadian effect; color quality; solid-state lighting; vision performance; white LED

\section{INTRODUCTION}

Over the decade, the solid-state lighting sources based on white lightemitting diodes (LEDs) has improved to overtake incandescent and fluorescent lighting types because of many advantages such as high brightness, ecofriendliness, long-life time, small size, low power consumption and fast response time. ${ }^{1,2}$ For these reasons, the LEDs can be applied indoor and outdoor lightings as well as for signals and in displays. In addition to the considerable impact of LEDs on energy consumption efforts, interest in the use of LED white lighting for human health has grown rapidly.

Recently, many bio- and medical-related research groups have reported that the spectral profiles of light emitted by white artificial lighting impacts our circadian physiology, alertness and cognitive performance levels. ${ }^{3,4}$ Several recent findings confirm that bright artificial light can be used in a number of therapeutic applications, such as in the treatment of seasonal affective disorder (SAD), ${ }^{5}$ certain sleep problems, ${ }^{6}$ depression, ${ }^{7}$ jet lag, ${ }^{8}$ sleep disturbances in those with Parkinson's disease, ${ }^{9}$ the health consequences associated with shift work ${ }^{10}$ and the resetting of the human circadian clock. ${ }^{11}$ However, quiet recently, Falchi et al. ${ }^{12}$ reported that artificial lighting changes natural processes, interferes with melatonin production and disrupts the circadian rhythm. It was also reported that blue light has a greater tendency than other colors to affect living organisms through the disruption of their biological processes which rely upon natural cycles of daylight and darkness. Consequently, exposure to blue light late in the evening and at night may be detrimental to one's health. ${ }^{3,13}$
Thus far, nearly all artificial lighting types, including white LEDs, have been optimized by characterizing a restricted number of figures of merit for good color quality and high vision performance, such as the correlated color temperature (CCT), color rendering index (CRI), luminous efficacy of radiation (LER) and luminous efficacy (LE). In a concerted view of the color, vision and circadian points, it is important to attain proper performance values in all figures of merit for healthy and smart lightings. Figure 1 summarizes all possible and important figures of merit pertaining to circadian performance as well as vision performance and the color quality of artificial lighting sources. Detailed explanations of the necessity of all figures of merit are included in the section on 'results and discussion'.

Previously, we developed a highly efficient $R_{B, M} A_{B, M} G_{B, M} B$ or $R_{B, M} A_{B, M} G_{B, M} C_{B}$ four-package white LED system in an effort to enhance $L E$ and CRI at several desired CCTs. ${ }^{14-16}$ Here, $R_{B, M} A_{B, M} G_{B, M}$, represents a long-wavelength pass dichroic filter (LPDF)-capped, monochromatic red/amber/green phosphor converted-LED (pcLED) pumped by a blue LED chip. B denotes an InGaN blue LED and $C_{B}$ denotes as a cyan phosphor partially-converted blue LED. ${ }^{14-16}$ In this study, we demonstrate all attainable figures of merit required for optimizing the circadian rhythm, vision performance and color quality of various $R_{B, M} A_{B, M} G_{B, M} B$ four-package white LEDs as well as commercialized lighting sources and daylight. We also optimize the optical properties of various $R_{B, M} A_{B, M} G_{B, M} B$ four-package white LEDs while varying the emission wavelength of each colored LED. All optimization processes are conducted to attain healthy, natural, 


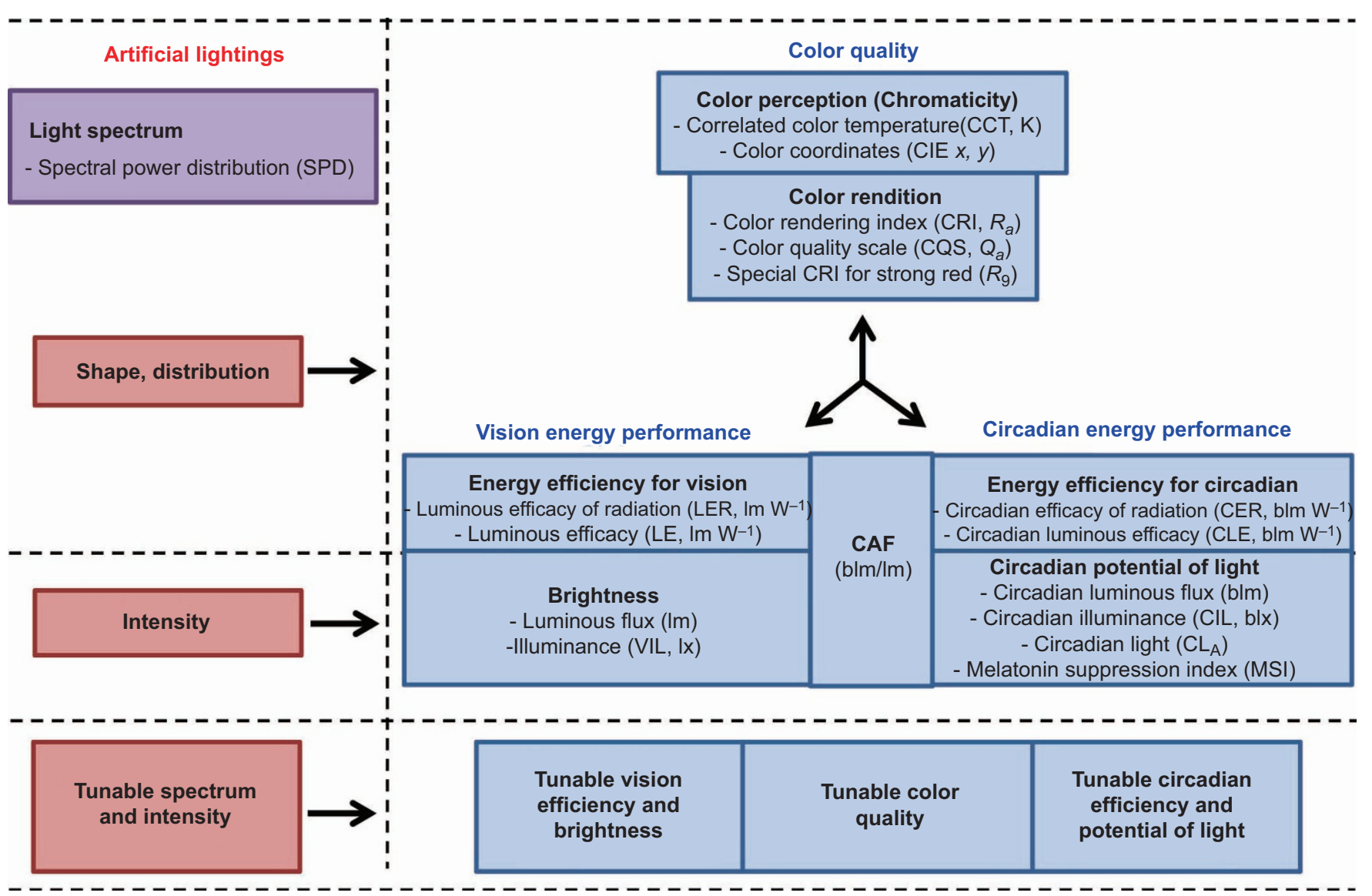

Figure 1 Schematic diagram of all possible figures of merit pertaining to circadian performance as well as vision performance and the color quality of artificial lighting.

efficient and tunable four-package white LEDs by comparing their figures of merit of daylight as well as presently commercialized light sources.

\section{MATERIALS AND METHODS}

Figure of merit for circadian performance, vision performance and color quality

By analyzing the spectral power distribution (SPD) of white light, the LER and the circadian efficacy of radiation (CER) values are respectively calculated and selected as the representative figures of merit for vision energy efficiency and circadian energy efficiency for any type of source. ${ }^{17}$

As previously reported, the LER and CER can be defined as the ratio of the luminous flux to the radiant flux $(S(\lambda))$ and the ratio of the circadian luminous flux to the radiant flux $(S(\lambda)),{ }^{17,18}$ respectively.

$$
\begin{gathered}
K=K_{0} \int_{380 \mathrm{~nm}}^{780 \mathrm{~nm}} V(\lambda) S(\lambda) / \int_{0}^{\infty} S(\lambda) \mathrm{d} \lambda \\
K_{c}=K_{c 0} \int_{380 \mathrm{~nm}}^{780 \mathrm{~nm}} C(\lambda) S(\lambda) / \int_{0}^{\infty} S(\lambda) \mathrm{d} \lambda
\end{gathered}
$$

where $V(\lambda)$ is the photopic spectral luminous efficiency function and $C(\lambda)$ is the circadian spectral sensitivity function. Among several suggested $C(\lambda) \mathrm{s}$ in previous reports, ${ }^{17-21}$ we used the circadian spectral sensitivity function from Gall et al. in this experiment. See more details about the definition and selection of $(C(\lambda))$ in the Supplementary Information 1-1. Compared to the maximum wavelength $V(\lambda)$ at $550 \mathrm{~nm}, \mathrm{C}(\lambda)$ peaks at about $460 \mathrm{~nm}$ (Figure 2a), as noted in previous publications based on data pertaining to the action spectrum for melatonin regulation; ${ }^{19,20} K_{0}$ is the maximal spectral luminous efficacy for vision and $K_{c 0}$ is the circadian efficacy value for a non-visual system $\left(K_{0}=683 \mathrm{~lm} \mathrm{~W} \mathrm{~W}^{-1}\right.$ and $K_{c 0}=683$ blm W ${ }^{-1}$, see Ref. 17), respectively. Therefore, LER and CER were clearly defined as parameters explaining how visually and non-visually bright the radiation of the emission spectrum are as perceived by the vision and non-vision system of the average human eye. Previous publications also proposed that the units for measuring circadian flux are the circadian lumen (cirlm) ${ }^{21}$ or biolumen (blm). ${ }^{17,22}$ Therefore, the proposed units of CER and $K_{c 0}$ are either circadian lumens or biolumens per watt (cirlm $\mathrm{W}^{-1}$ or blm $\mathrm{W}^{-1}$, respectively).

As previously reported, the circadian action factor (CAF), another figure of merit, is defined as the ratio CER to LER. ${ }^{17,22}$ Simply, this refers to the biological action per unit of visual response. Different CAF values are advisable depending on the time of day: high CAF during daytime and low CAF during nighttime. CAF values can be used as an optimizing tool for artificial light for human health.

The circadian luminous efficacy (CLE) is defined from the equation (4), explaining how brightly the non-visual light is emitted from an artificial lighting system.

$$
\begin{gathered}
\operatorname{CAF}\left(\operatorname{blm~lm}{ }^{-1}\right)=\operatorname{CER}\left(\operatorname{blm~W} \mathrm{W}^{-1}\right) / \operatorname{LER}\left(\operatorname{lm~W^{-1}}\right) \\
\operatorname{CLE}\left(\operatorname{blm~W^{-1}}\right)=\operatorname{LE}\left(\operatorname{lm~} \mathrm{W}^{-1}\right) \times \operatorname{CAF}\left(\operatorname{blm~lm}^{-1}\right)
\end{gathered}
$$

To determine the minimum amount of white light from individual artificial light sources to activate the non-visual system, it is also 

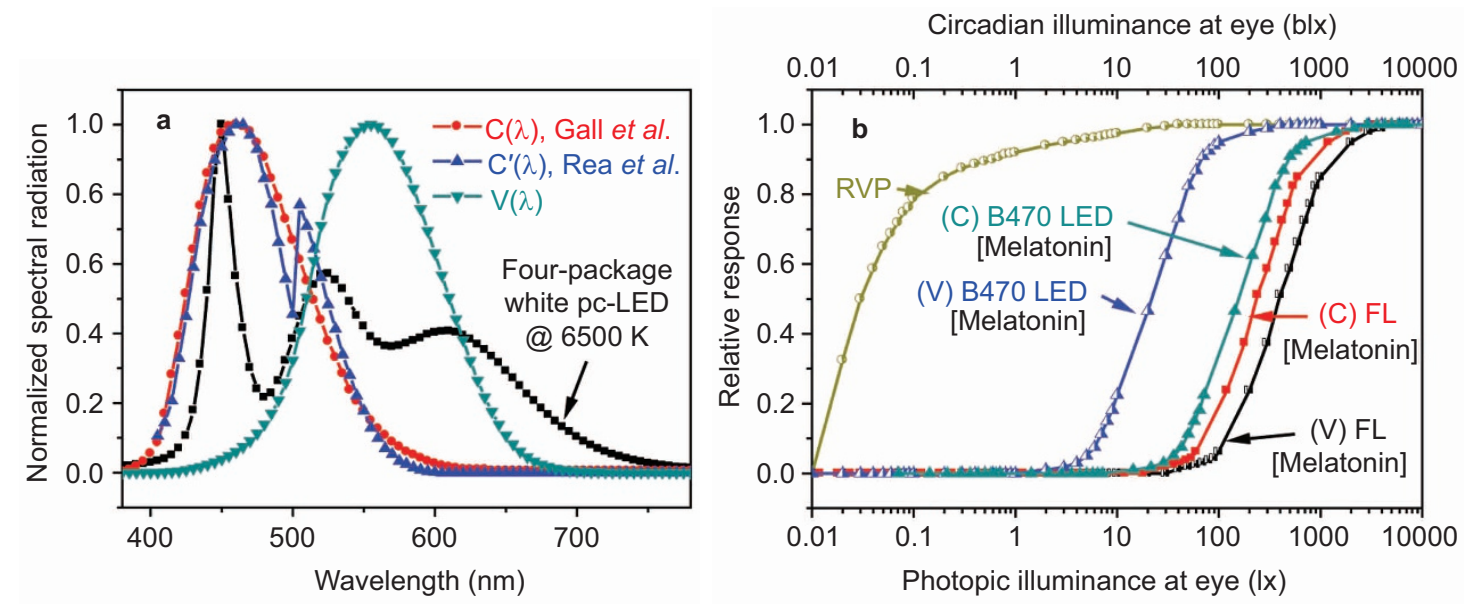

Figure 2 (a) The normalized spectra of the circadian spectral sensitivity function $\left(C(\lambda)\right.$ Gall et al. ${ }^{18}$ and $C^{\prime}(\lambda)$ Rea et al. $\left.{ }^{21}\right)$, the photopic spectral luminous efficiency function $(V(\lambda))$ and the normalized spectrum of four-package white pc-LED (B450-G515-A590-R640) at $6500 \mathrm{~K}$. (b) The RVP and relative melatonin suppression value as a function of the visual illuminance from a $4100 \mathrm{~K}$ full-spectrum FL ((V) FL) and a $470 \mathrm{~nm}$ blue LED ((V) B470 LED) at the eye, and the relative melatonin suppression value as a function of circadian illuminance from a $4100 \mathrm{~K}$ full-spectrum FL ((C) FL) and a $470 \mathrm{~nm}$ blue LED ((C) B470 LED) at the eye. FL, fluorescent lamp; LED, light-emitting diode; pc-LED, phosphor-converted LED; RVP, relative visual performance.

important to measure the circadian illuminance (CIL) of the lighting instead of relying on the visual illuminance (VIL) value. Similar to the illuminance measurement method, the CIL can be obtained by measuring the total circadian luminous flux incident on a surface per unit area. Simply, the CIL can be defined from the equation below:

$$
\begin{aligned}
& \operatorname{CIL}(\text { blx })=\operatorname{CAF}\left(\operatorname{blm~lm}^{-1}\right) \times \operatorname{VIL}(1 \mathrm{x})= \\
& \left(\operatorname{CER}\left(\operatorname{blm~W} \mathrm{W}^{-1}\right) / \operatorname{LER}\left(\operatorname{lm~} \mathrm{W}^{-1}\right)\right) \times \operatorname{VIL}(1 \mathrm{x})
\end{aligned}
$$

If we know the CAF of any type of lighting and the VIL, we can calculate the CIL of white light under a specified condition. Furthermore, the lamp spectra, which can have a high CAF value, have the potential to concentrate the energy in a spectral region that is circadian-sensitive, thus having a strong impact on human health.

Due to the introduction of figures of merit for the circadian system, it becomes possible to use CIL to determine a lamp's capability to activate the circadian system. Figure $2 \mathrm{~b}$ shows the relative visual performance and relative melatonin suppression value as a function of the VIL from full-spectrum fluorescent lamps (FLs) and blue LEDs ${ }^{21}$ at the eye as reported in previous publications. Both the melatonin suppression curves of the full-spectrum FL lamp and a $470 \mathrm{~nm}$ blue LED are located at the different illuminance levels $(\sim 15$ times of difference). As mentioned above, this figure simply indicates that the VIL is not the proper scale to determine the threshold amount of light to stimulate the circadian photobiological system. Accordingly, the CIL was introduced as a measure of how an artificial light source affects the circadian photobiological system. With the CAF and VIL of any type of lighting, in Equation (5), the CIL of white light can be calculated under a specified condition. The calculated melatonin suppression curves as a function of the CIL from both a full-spectrum FL lamp and a $470 \mathrm{~nm}$ blue LED are added in Figure 2b. On the basis of the SPDs of light sources, it was assumed that the CAF values of the full-spectrum FL lamp and $470 \mathrm{~nm}$ blue LED are $\sim 0.6$ and $\sim 7.2$, respectively, in this calculation. As a result, two melatonin suppression curves as a function of the CIL converge to the middle region between both melatonin suppression curves as a function of the VIL. Consequently, if the threshold levels for the circadian system are measured on a CIL scale, the health effect of individual lamps can be analyzed by the figures of merit for the circadian system, in this case, the CER, CLE and CAF. These results can resolve the large deviation problem of the VIL threshold for melatonin suppression between different types of artificial lamps.

Other figures of merit for the circadian system have been reported such as circadian light value $\mathrm{e}^{23-25}\left(\mathrm{CL}_{\mathrm{A}}\right)$ and melatonin suppression index ${ }^{26}$ (MSI) to evaluate the circadian potential of light sources. The MSI shows the potential spectral impact of a lamp SPD on the melatonin suppression. See more details in Supplementary Information $1-2$.

The CCT is commonly used to describe the perceived color of white artificial light sources, but CRI and color quality scale (CQS) are quantitative measures of the capability of a light source to reproduce the true colors of illuminated objects in comparison with an ideal light source. $^{27-29}$

Thus far, CRI has been widely used in the lighting society to demonstrate how well a light source can reproduce colors, but a high CRI cannot guarantee good saturated colors of illuminated objects ${ }^{30}$ because the CRI was calculated with only the first eight $\left(R_{1}-R_{8}\right)$ values of CIE from the recommended 14 test color samples for the calculation of the CRI. ${ }^{31}$ Therefore, to achieve a high-quality color rendition of light source, the strong red $\left(R_{9}\right)$ value is needed.

Recently, the CQS was proposed as a new color rendition metric to compensate for the insufficient capability of the CRI to reproduce saturated colors. ${ }^{27,29,31-33}$ The CQS is calculated using 15 reflective Munsell samples and a saturation factor; moreover, it becomes more effective when an artificial light source enhances the object chroma. These figures of merit can be calculated from the measured SPDs of real white LEDs while varying the applied current of each colored LED.

\section{Fabrication of LPDF-capped red/amber/yellow/green pc-LEDs and partially-converted cyan pc-LED ${ }^{14,16}$}

The explanations of fabrication and characterization of the LPDFs in Supplementary Information 2. To fabricate LPDF-capped red/amber/ yellow/green full-down converted monochromatic pc-LEDs and partially converted cyan pc-LED, an InGaN blue LED $\left(\lambda_{\max }=445 \mathrm{~nm}\right)$ was used as an excitation source for various color phosphors of pc-LEDs. The blue LED chips were purchased from Dongbu LED, Inc. (Yongincity, Korea). Optimum amounts of each color phosphor were 
dispersed in a silicone binder to create a phosphor paste, and the same amounts of resulting phosphor pastes were dropped onto a cup-type blue LED to create each colored pc-LED. After the phosphor paste was dropped onto a blue LED, the paste was hardened by heating in each case. The LPDFs (L535 for yellow/green and L550 for red/amber) were capped on top of the pc-LEDs with an air gap to realize full downconverted pc-LEDs. The green/amber/red LEDs were referred to according to their colors (e.g., red LED: R, amber LED: A and green LED: G); the two types of blue LEDs were named based on their color and wavelength (B450 and B460); and the pc-LEDs were named based on their color and wavelength (e.g., a LPDF-capped green pc-LED with an emitting wavelength of $520 \mathrm{~nm}$ : G520). Supplementary Tab. S1 shows the optical properties of each colored LED and the pc-LEDs along with the chemical formula of the phosphors used in each colored LED and pc-LEDs.

Characterization of LPDF-capped red/amber/yellow/green pcLEDs, partially converted cyan pc-LED and $R_{B, M} A_{B, M} G_{B, M} B$ fourpackage white LEDs $^{14,16}$

The emission spectra and luminous flux of the forward emission from LPDF-capped red/amber/yellow/green pc-LEDs, a cyan pc-LED and red/amber/green/blue semiconductor-type LEDs were measured in an integrated sphere using a spectrophotometer Darsarpro-5000; PSI
Co., Ltd (Suwon-city, Korea) with an applied current of $60 \mathrm{~mA}$. The SPDs and luminous flux of the $R_{B, M} A_{B, M} G_{B, M} B$ four-package white LED systems were put into a square lattice fixture and were measured in an integrated sphere using a spectrophotometer Darsarpro-5000; PSI Co., LTD (Suwon-city, Korea) while controlling the applied current of each primary LED with a total applied current of $240 \mathrm{~mA}$. A set of four-package pc-LEDs (R640, A590, G520, B450) was selected for the reference primary LEDs to compare the optical properties, i.e., the circadian and vision performance levels and the color qualities with a set of four CCTs $(10000 \mathrm{~K}, 6500 \mathrm{~K}, 3500 \mathrm{~K}$ and $2000 \mathrm{~K})$ while changing each colored pc-LED.

\section{RESULTS AND DISCUSSION}

Optical properties of daylight with the daily time period and various artificial lighting types

Many scientists still consider it to be their goal to realize daylight spectra from artificial lighting sources, including LED lightings.

We measured and calculated the figures of merit of daylight and artificial lightings in order to know the requirements for mimicking daylight. All characterizations of daylight were carried out on the top of a five-story science building at Kookmin University in Seoul, Korea (15 May 2013, sunny, sunrise: 5:23 and sunset: 19:34). Figure 3 shows the changes of the SPDs, and the VIL, CIL, CCTs, LER, CER and CAF
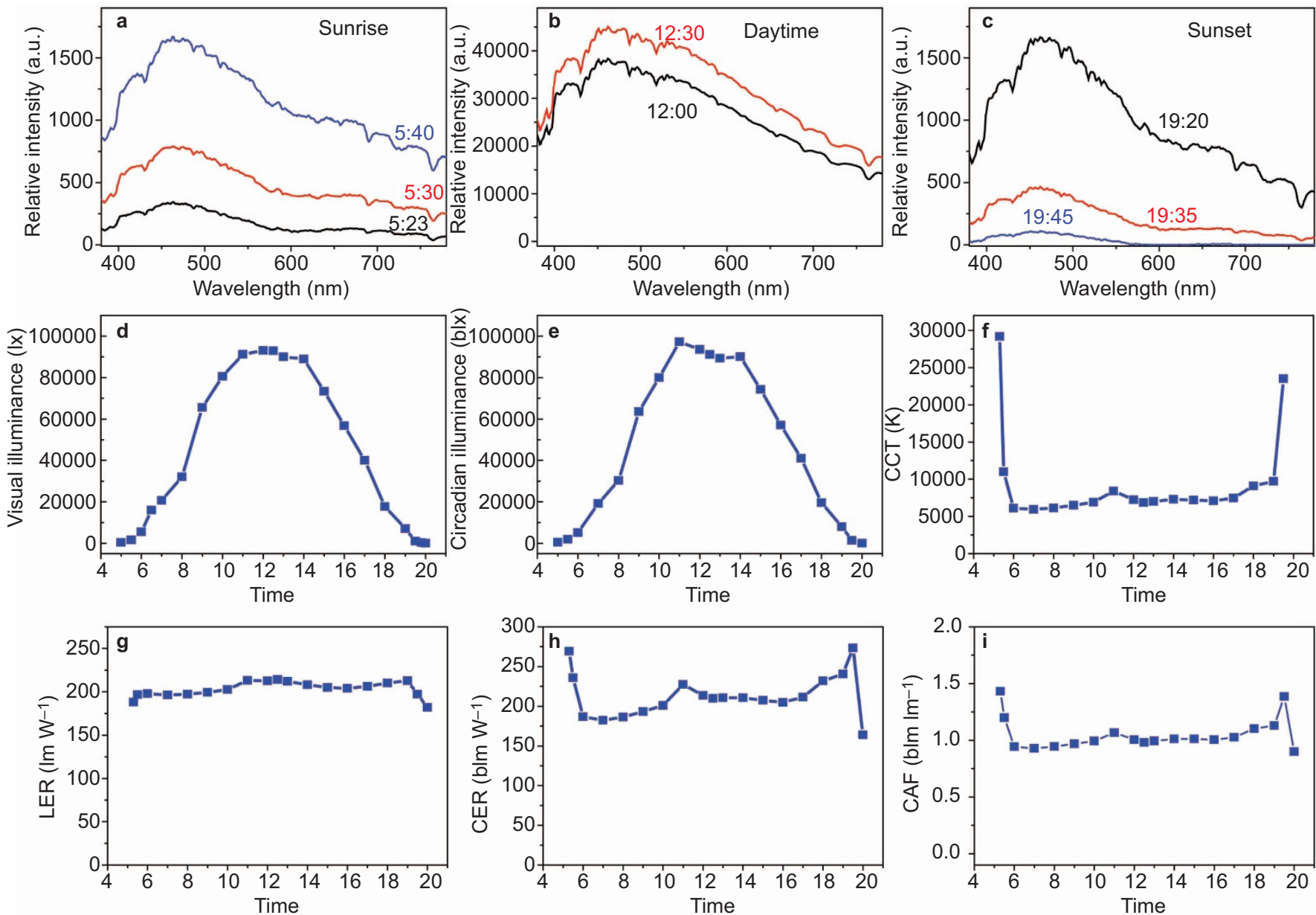

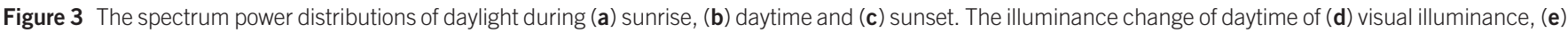

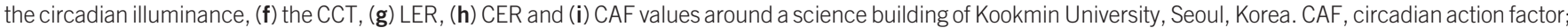
CCT, correlated color temperature; CER, the circadian efficacy of radiation; LER, luminous efficacy of radiation. 
values of daylight calculated from the SPDs of daylight measured every hour from morning to evening. The amounts of visual and non-visual light from the sun, those are, VIL and CIL, simply increase from early in the morning until 12:30 p.m. in the afternoon and then simply decrease until sunset (Figure $3 \mathrm{a}-\mathrm{c}$ ). Figure $3 \mathrm{i}$ shows that the CAF values of daylight remain nearly constant near $\sim 1.0$. This indicates that the circadian brightness of daylight is similar to the value of the visual brightness of daylight.

The characteristics of artificial lighting types that are ideal for vision are fairly different from than those that are maximally effective for the circadian system. ${ }^{19}$ The most general indoor and outdoor lighting sources used in the present market were developed exclusively for improving the vision performance and color quality by considering the energy saving and a CCT mimicking of daylight.

Figure 4 and Table 1 show the reported or measured SPDs and figures of merit of 10 commercialized types of lighting and two types of four-package white LEDs. These data indicate that the SPDs of all 10 artificial lighting types used presently indoors and outdoors are fixed with a specific CCT. As shown in the artificial lighting performance data in Figure 4 and Table 1, there is no ideal lamp to meet all of the requirements to attain high vision performance and good color quality, while also having a highly positive health effect. Because some of figures of merit for artificial lighting sources are in a tradeoff relationship, managing the light of an artificial lighting source to optimize the vision performance, color quality and non-visual health effect
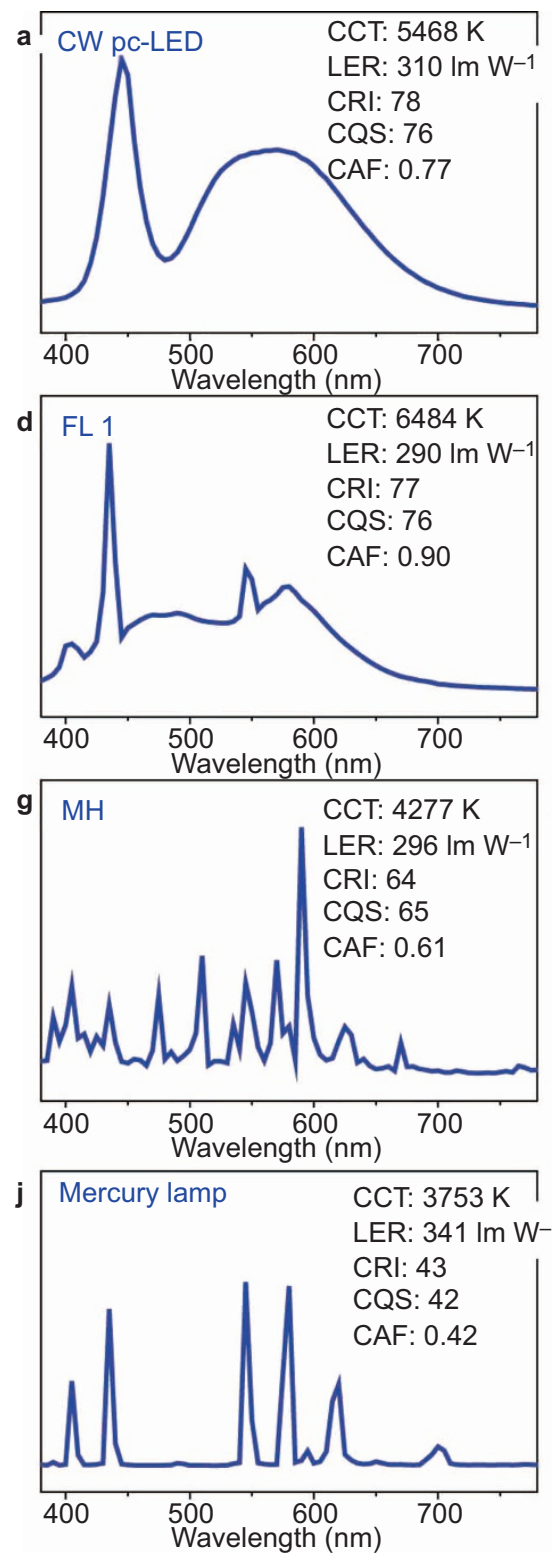
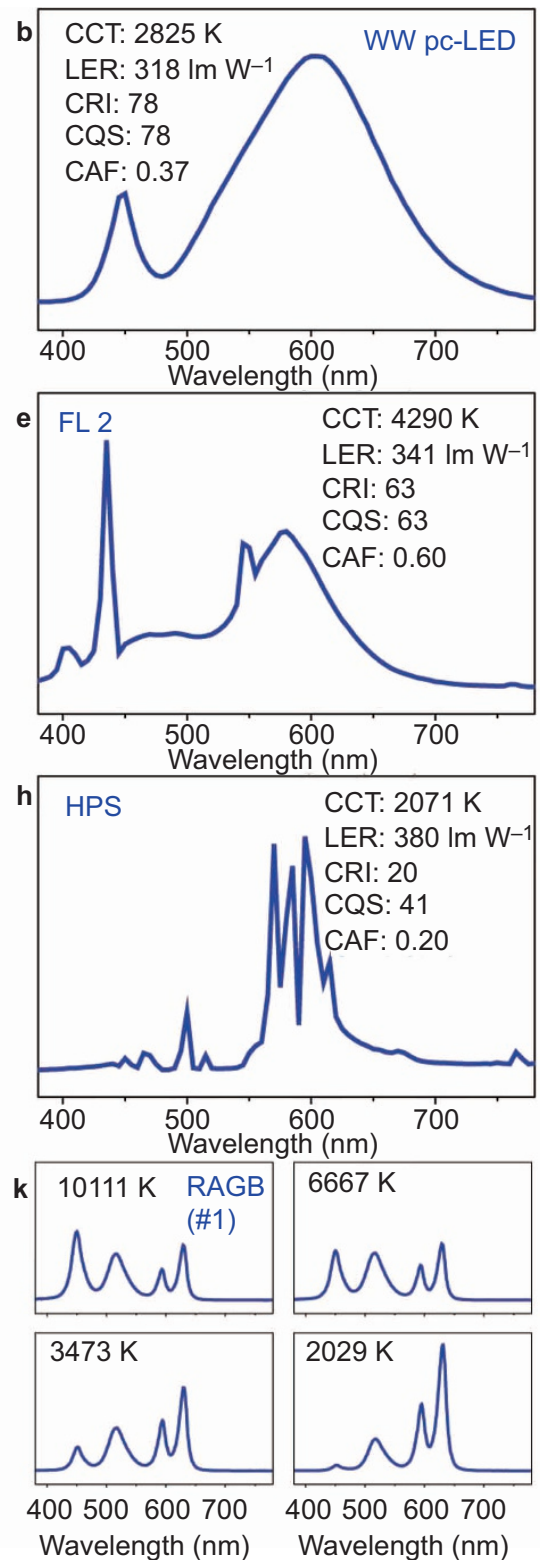
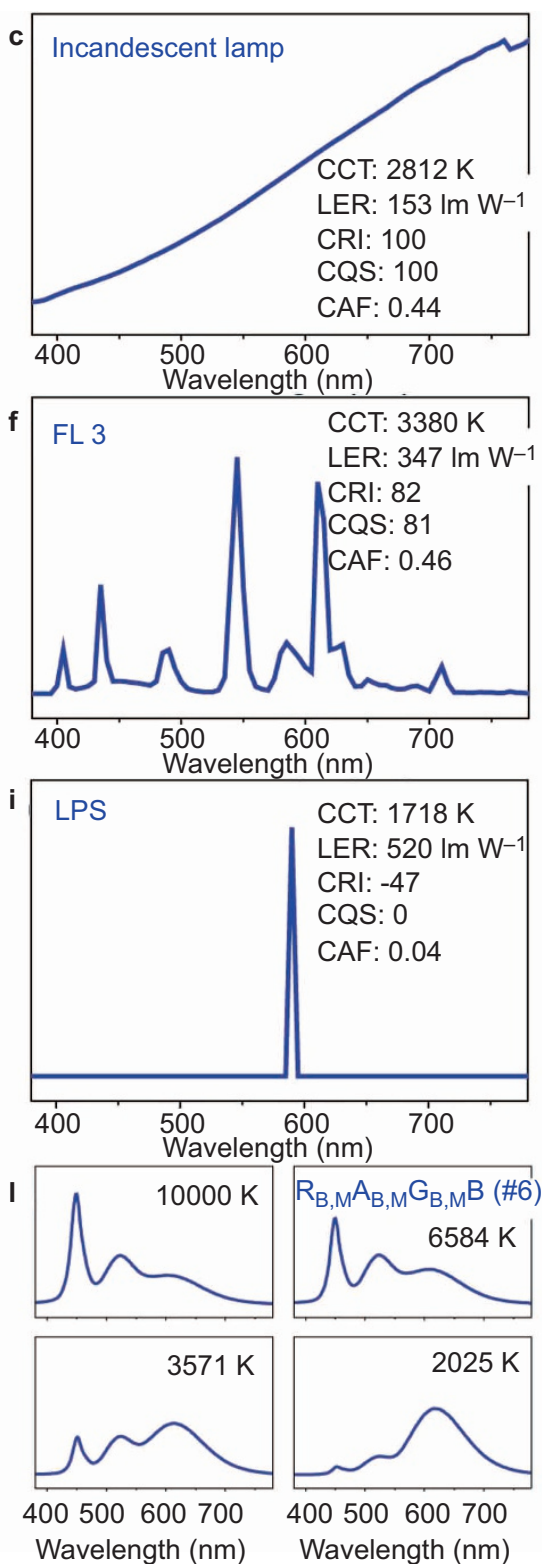

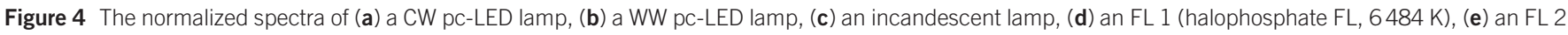

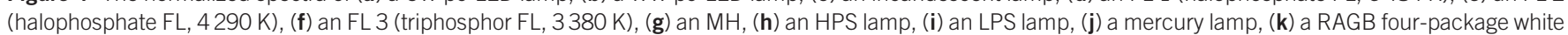

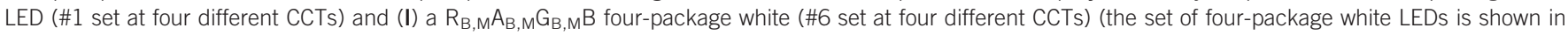

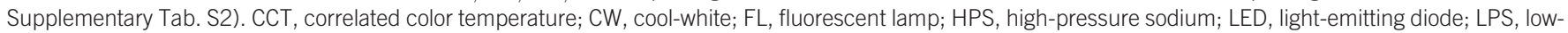
pressure sodium; MH, metal-halide; pc-LED, phosphor-converted LED; WW, warm-white. 
Table 1 The figures of merits of 10 types of widely commercialized artificial lamps and two types of four-package white LEDs at different CCTs

\begin{tabular}{|c|c|c|c|c|c|c|c|c|c|c|}
\hline & & CCT $(\mathrm{K})$ & $\mathrm{CRI}\left(R_{a}\right)$ & $\operatorname{CQS}\left(Q_{a}\right)$ & $\operatorname{LE}\left(\operatorname{Im~W} W^{-1}\right)$ & 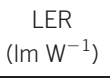 & 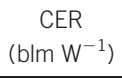 & 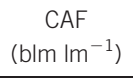 & MSI & $\begin{array}{l}\text { Illuminance (Ix for } \\
250 \mathrm{blx} \text { ) }\end{array}$ \\
\hline a & CW pc-LED & 5468 & 78 & 76 & 98 & 310 & 238 & 0.77 & 0.70 & 325 \\
\hline$b$ & WW pc-LED & 2825 & 78 & 78 & 98 & 318 & 117 & 0.37 & 0.30 & 676 \\
\hline c & Incandescent & 2812 & 100 & 100 & $26^{a}$ & 153 & 67 & 0.44 & 0.36 & 568 \\
\hline d & FL-1 & 6484 & 77 & 76 & $55-70^{b}$ & 290 & 261 & 0.90 & 0.87 & 278 \\
\hline e & FL-2 & 4290 & 63 & 63 & $55-70^{b}$ & 341 & 203 & 0.60 & 0.57 & 417 \\
\hline$f$ & FL-3 & 3380 & 82 & 81 & $55-70^{\mathrm{b}}$ & 347 & 159 & 0.46 & 0.41 & 543 \\
\hline g & $\mathrm{MH}$ & 4277 & 64 & 65 & $65-115^{a}$ & 296 & 182 & 0.61 & 0.74 & 410 \\
\hline h & HPS & 2071 & 20 & 41 & $100-140^{a}$ & 380 & 75 & 0.20 & 0.15 & 1250 \\
\hline i & LPS & 1718 & -47 & 0 & $100-200^{b}$ & 431 & 18 & 0.04 & 0.02 & 6250 \\
\hline j & Mercury lamp & 3753 & 43 & 42 & $60^{\mathrm{a}}$ & 341 & 144 & 0.42 & 0.51 & 595 \\
\hline \multirow[t]{4}{*}{ k } & $\mathrm{RAGB}^{\mathrm{C}}$ & 10111 & 69 & 73 & 54 & 261 & 326 & 1.25 & 1.13 & 200 \\
\hline & & 6667 & 74 & 73 & 56 & 290 & 290 & 1.00 & 0.88 & 250 \\
\hline & & 3473 & 72 & 70 & 65 & 322 & 196 & 0.61 & 0.52 & 411 \\
\hline & & 2029 & 70 & 72 & 75 & 327 & 100 & 0.31 & 0.24 & 816 \\
\hline \multirow[t]{4}{*}{ I } & $\mathrm{R}_{\mathrm{B}, \mathrm{M}} \mathrm{A}_{\mathrm{B}, \mathrm{M}} \mathrm{G}_{\mathrm{B}, \mathrm{M}} \mathrm{B}^{\mathrm{d}}$ & 10000 & 92 & 92 & 89 & 262 & 304 & 1.16 & 1.03 & 216 \\
\hline & & 6584 & 94 & 94 & 96 & 280 & 258 & 0.92 & 0.81 & 271 \\
\hline & & 3437 & 96 & 96 & 99 & 294 & 156 & 0.53 & 0.44 & 470 \\
\hline & & 2025 & 84 & 89 & 83 & 271 & 69 & 0.25 & 0.19 & 983 \\
\hline
\end{tabular}

Abbreviations: CAF, circadian action factor; CCT, correlated color temperature; CER, the circadian efficacy of radiation; CQS, color quality scale; CRI, color rendering index; CW, cool-white; FL, fluorescent lamp; HPS, high-pressure sodium; LE, luminous efficacy; LPS, low-pressure sodium; LER, luminous efficacy of radiation; MH, metal-halide; MSI, melatonin suppression index; pc-LED, phosphor-converted LED; WW, warm-white.

${ }^{\text {a }}$ Philips product catalog (USA).

b M F Gendre, 'Two centuries of electric light source innovations' (2003).

Available from: http://www.einlightred.tue.nl/lightsources/history/light_history.pdf

${ }^{\mathrm{C}}$ RAGB semiconductor-type four-package white LED was calculated and measured in an integrated sphere with a total applied current of $240 \mathrm{~mA}$ (\#1 set in this experiment; see details from Supplementary Tab. S2).

${ }^{d} R_{B, M} A_{B, M} G_{B, M} B$ four-package white LED was calculated and measured in an integrated sphere with a total applied current of $240 \mathrm{~mA}$ (\#6 set in this experiment, $R 640$, A590, G515 and B450; see details from Supplementary Tab. S2).

becomes critical. Figure $4 \mathrm{k}$ and 41 show the SPDs of different types of tunable four-package white LEDs with various CCTs. These fourpackage white LEDs can control the figures of merit of light. The $\mathrm{R}_{\mathrm{B}, \mathrm{M}} \mathrm{A}_{\mathrm{B}, \mathrm{M}} \mathrm{G}_{\mathrm{B}, \mathrm{M}} \mathrm{B}$ tunable four-package white LED (Figure 41 and Table 1l) shows higher vision performance and color quality than RAGB tunable four-package white LED (Figure 4k and Table 1k). Therefore, it is clear that maintaining the capability of controlling all figures of merit of light is the most important prerequisite for good healthy and smart lighting systems. The detailed characterization method of daylight and the artificial lightings are in Supplementary Information 3.

Optical properties of long-wavelength pass dichroic filter (LPDF)capped pc-LEDs and blue LEDs

Figure 5a-d show the normalized electroluminescence spectra, color coordinates and photographs of five semiconductor-type LEDs (two blue, one green, one amber, one red), fifteen different colors of fullconverted, LPDF-capped pc-LEDs (six green, four amber-yellow, five red), which fabricated by simply capping of LPDF on top of the InGaN blue LED with each corresponding phosphor, ${ }^{34,35}$ and one partially converted cyan phosphor-coated blue LED.

Here, all monochromatic LEDs are denoted as a combination of the color and peak wavelength of the emission spectrum. The emission spectrum of all monochromatic pc-LEDs is well matched with that of the corresponding phosphors, as shown in Supplementary Fig. S3 and Tab. S1. ${ }^{35}$

All CERs, LERs. CLEs, LEs and CAFs of monochromatic colored LEDs for both the visual and non-visual performance are measured at $60 \mathrm{~mA}$ (rated current) and displayed with the change of the peak wavelength of the monochromatic LEDs in Figure 5e-g, and Supplementary Tab. S1. The figures indicate that the shapes of the dependent graphs of LER and LE on the peak wavelength are identical in appearance to the photopic spectral luminous efficiency function (photopic sensitivity) and the trends of CER and CLE with the peak wavelength resemble the wavelength dependence of the circadian spectral sensitivity function (Figures 2 and 5). The figures also confirm that bluish LEDs have higher CAF values (the ratio of CER to LER) and that the reddish LEDs have lower CAF values. This indicates that bluish LEDs are more effective than the reddish LEDs on the circadian system of humans. Therefore, the use of bluish LEDs, as well as all high CAF sources, should be limited after sunset.

Optical properties of $R_{B, M} A_{B, M} G_{B, M} B$ four-package white LEDs for optimizing the vision performance, color quality and circadian performance

In this study, we compare the optical properties of the conventional RAGB multipackage approach and $\mathrm{R}_{\mathrm{B}, \mathrm{M}} \mathrm{A}_{\mathrm{B}, \mathrm{M}} \mathrm{G}_{\mathrm{B}, \mathrm{M}} \mathrm{B}$ multi-package approach by comparing the figures of merit for vision, circadian performances and color qualities at different CCTs. Although the conventional RAGB multipackage approach allows for the facile dynamic control of color points and provides high LER, this approach has several disadvantages, such as the low efficiency of green and amber LEDs (the 'green gap' problem), the different temperature/current/ time dependence of each colored LED and the reduced color quality of the light due to the narrow-band spectrum. These shortcomings inhibit the wider penetration of these RAGB multipackage white LEDs in lighting applications. ${ }^{36,37}$ 

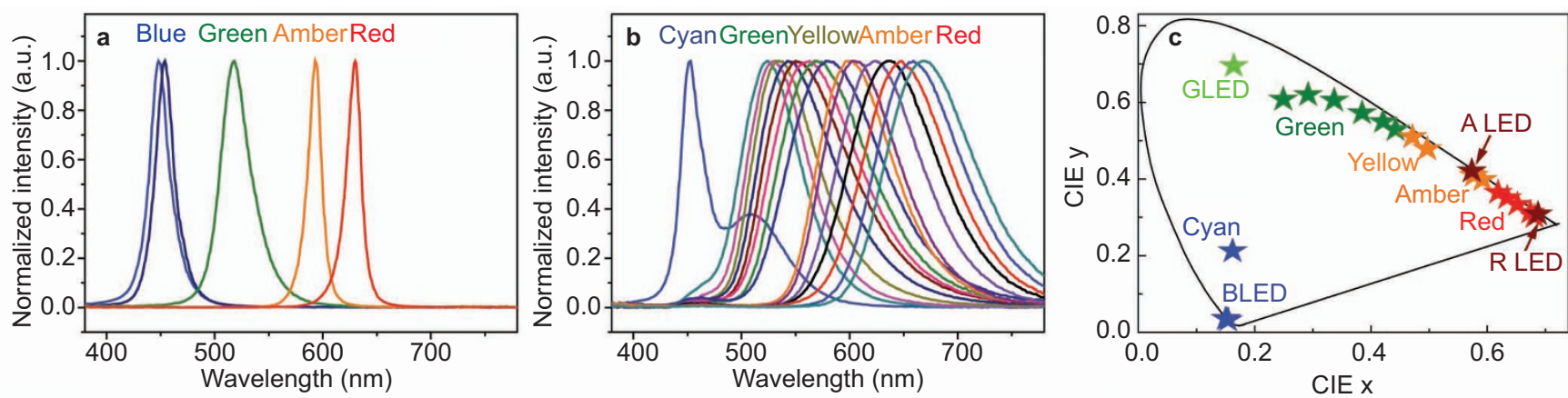

d

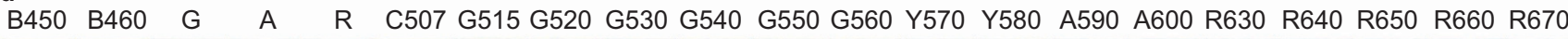
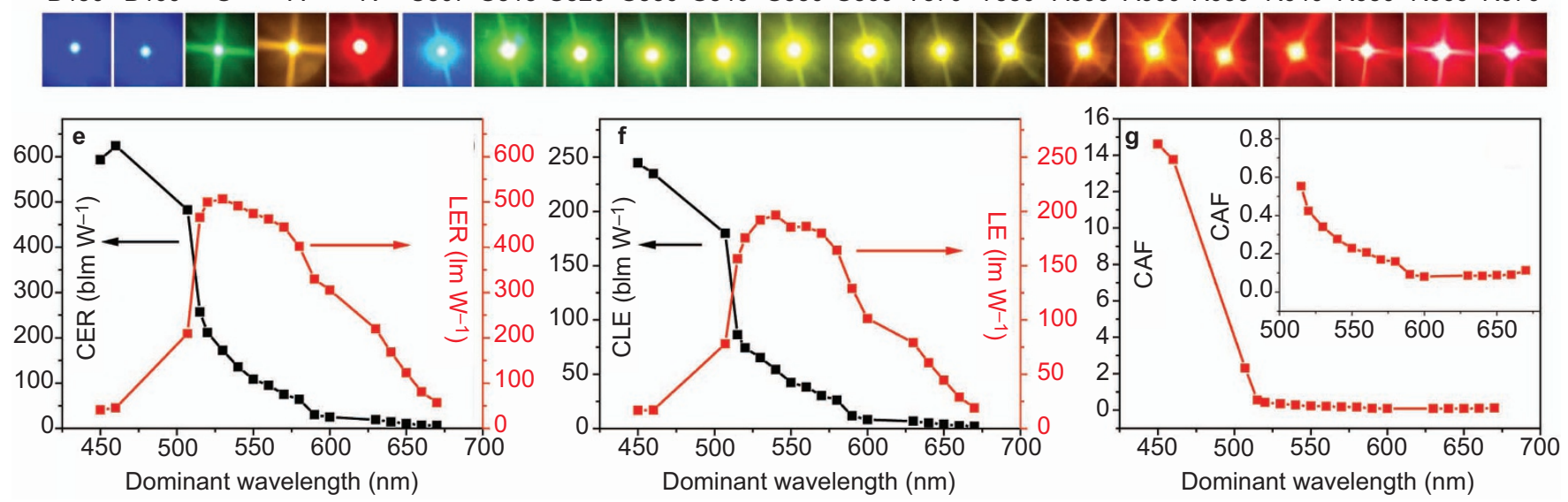

Figure 5 The normalized electroluminescence spectra of (a) blue, green, amber and red semiconductor-type LEDs and (b) a partially converted cyan phosphorcoated blue LED and full-down converted, LPDF-capped pc-LEDs. (c) The 1931 CIE color coordinates of B/G/A/R semiconductor-type LEDs, partially converted cyan phosphor-coated blue LED and full-down converted LPDF-capped pc-LEDs. (d) Images of B/G/A/R semiconductor-type LEDs, a partially converted cyan phosphorcoated blue LED and full-down converted LPDF-capped pc-LEDs. (e) CER and LER, (f) CLE and LE and (g) CAF as a function of the peak wavelength of 15 different colors of full-converted, LPDF-capped pc-LEDs, one partially converted cyan phosphor-coated blue LED and two InGaN blue LEDs. CAF, circadian action factor; CER, the circadian efficacy of radiation; CIE, color coordinates; CLE, circadian luminous efficacy; LE, luminous efficacy; LED, light-emitting diode; LER, luminous efficacy of radiation; LPDF, long-wavelength pass dichroic filter; pc-LED, phosphor-converted LED.

In this experiment, two RAGB multi-package white LEDs using two different blue LEDs $\left(\lambda_{\max }=450\right.$ and $\left.460 \mathrm{~nm}\right)$ were characterized as standard samples for comparison. Furthermore, we selected a set of primary LEDs with peak wavelengths of $640 \mathrm{~nm}$ (red, R640), $590 \mathrm{~nm}$ (amber-yellow, A590), $520 \mathrm{~nm}$ (green, G520) and $450 \mathrm{~nm}$ (blue, B450) for the $R_{B, M} A_{B, M} G_{B, M} B$ four-package white LEDs as shown in the schematic diagram of the $R_{B, M} A_{B, M} G_{B, M} B$ four-package white LEDs in Figure 6a. We also characterized and compared the figures of merit of various $R_{B, M} A_{B, M} G_{B, M} B$ four-package white LEDs in terms of the vision performance, circadian performance and color quality with a change of the peak wavelength of each colored series (Supplementary Tab. S2 shows the LED combinations of the $R_{B, M} A_{B, M} G_{B, M} B$ fourpackage white LED set).

Based on the emission spectrum of each four-package white LED, meaningful figures of merit of all types of $R_{B, M} A_{B, M} G_{B, M} B$ LEDs in white-light applications, in this case, the LE, LER, CAF, CLE, CER, CQS, CRI and $R_{9}$ values were calculated and summarized, as displayed in Figure 6b-i at four CCT values ( $10000 \mathrm{~K}, 6500 \mathrm{~K}$ (daylight), $3500 \mathrm{~K}$ (warm white) and $2000 \mathrm{~K}$ (firelight)). Among the 20 different fourpackage white LEDs shown in Supplementary Tab. S2 and Figure 6, the best color quality, the highest vision performance and an excellent circadian effect are attained from the different combination of a $R_{B, M}, A_{B, M}, G_{B, M}$ and $B$ LED in four-package white LED. In this figure, a combined four-package LED of R640, A590, G520 and C507 is selected as having the best color quality, while a combined four-package LED of R640, A590, G540 and B450 is chosen as having the best LE and CLE. Although one $R_{B, M} A_{B, M} G_{B, M} B$ combination cannot show the best performance in all figures of merit for the color quality, vision performance and circadian effect, many of the $\mathrm{R}_{\mathrm{B}, \mathrm{M}} \mathrm{A}_{\mathrm{B}, \mathrm{M}} \mathrm{G}_{\mathrm{B}, \mathrm{M}} \mathrm{B}$ combinations have better color quality, higher vision performance and a more tunable circadian effect than any commercialized artificial lighting sources. For example, a four-package white LED that combines R640, A590, G515 and B450 offers excellent color qualities (CRI>94, CQS $>94$ and $R_{9}>70$ ), excellent vision performances (LER $>$ $\left.280 \mathrm{~lm} \mathrm{~W}^{-1}, \mathrm{LE}>95 \mathrm{~lm} \mathrm{~W}^{-1}\right)$ and a highly tunable circadian effect $\left(\mathrm{CER}=156\right.$ and $258 \mathrm{blm} \mathrm{W}^{-1}, \mathrm{CLE}=52$ and $88 \mathrm{blm} \mathrm{W}^{-1}, \mathrm{CAF}=0.53$ and 0.92 ) at cool- and warm-white CCTs of $6500 \mathrm{~K}$ and $3500 \mathrm{~K}$. In addition, both blue- and red-enriched emission (10000 $\mathrm{k}$ and $2000 \mathrm{~K}$ ) of $R_{B, M} A_{B, M} G_{B, M} B$ (R640, A590, G515 and B450) LEDs provide reasonable color qualities (CRI $=92$ and $84, \mathrm{CQS}=92$ and 89 and $R_{9}>90$ and 21), good vision performances (LER $>262 \mathrm{~lm} \mathrm{~W}^{-1}, \mathrm{LE}>$ $\left.83 \mathrm{~lm} \mathrm{~W}^{-1}\right)$ and wider tunable circadian effects $(\mathrm{CER}=304$ and $69 \mathrm{blm} \mathrm{W}^{-1}, \mathrm{CLE}=103$ and $22 \mathrm{blm} \mathrm{W}^{-1}, \mathrm{CAF}=1.16$ and 0.25 at CCTs of $10000 \mathrm{~K}$ and $2000 \mathrm{~K}$ ). Wide-band $\mathrm{R}_{\mathrm{B}, \mathrm{M}} \mathrm{A}_{\mathrm{B}, \mathrm{M}} \mathrm{G}_{\mathrm{B}, \mathrm{M}} \mathrm{B}$ four-package white LEDs can be considered as a possible artificial lighting candidates to mimic daylight reduce the blue portion of white light at night, and realize high vision performances, excellent color qualities and controllable circadian effects (Supplementary Figs. S5-S9). 
a
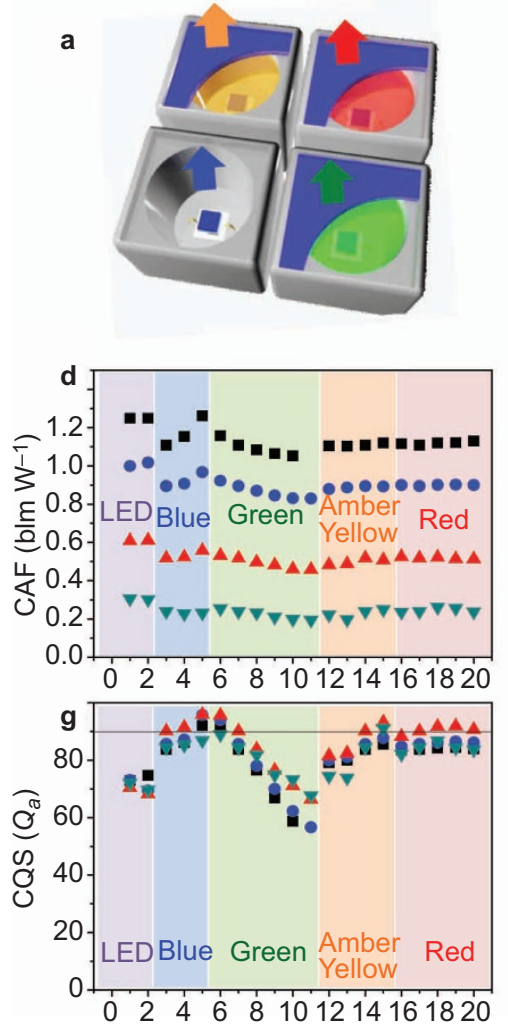
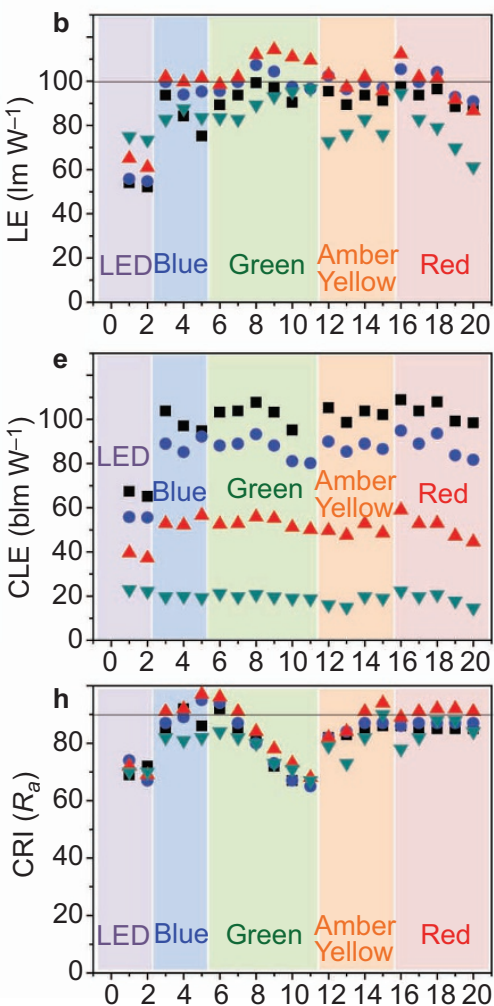

-10000K • 6500K $\Delta 3500 \mathrm{~K} \nabla 2000 \mathrm{~K}$
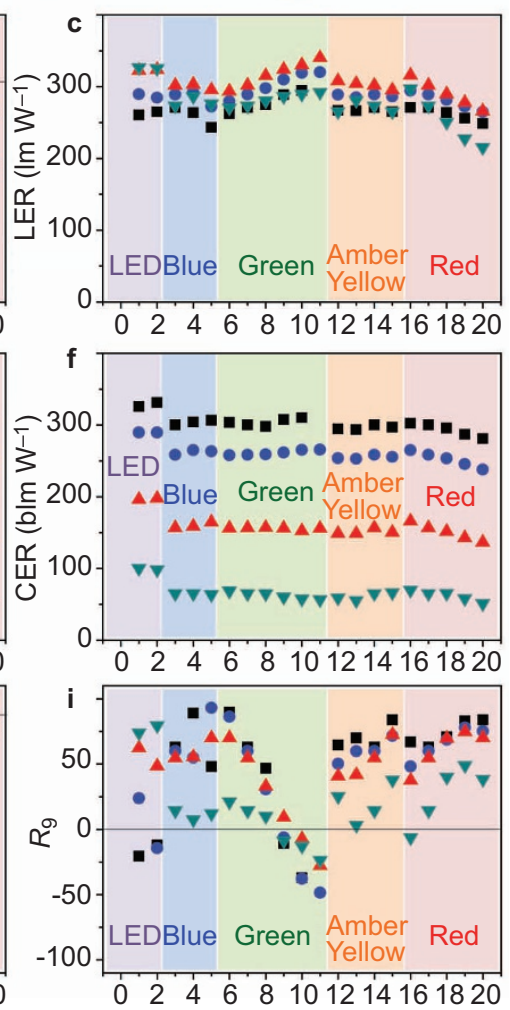

Figure 6 (a) A schematic diagrams of the $R_{B, M} A_{B, M} G_{B, M} B$ four-package white $L E D$. The eight figures of merits of all $R_{B, M} A_{B, M} G_{B, M} B$ four-package white $L E D$ and $R A G B$ white LED at $10000 \mathrm{~K}(\boldsymbol{\bullet}), 6500 \mathrm{~K}(\bullet$, cool white), $3500 \mathrm{~K}(\boldsymbol{\Delta}$, warm white) and $2000 \mathrm{~K}$ ( $\mathbf{\nabla}$, firelight). (b) LE, (c) LER, (d) CAF, (e) CLE, (f) CER, (g) CQS, (h) CRI and (i) $R$. CAF, circadian action factor; CER, the circadian efficacy of radiation; CLE, circadian luminous efficacy; CQS, color quality scale; CRI, color rendering index; CRI, clolor rendering index; LE, luminous efficacy; LED, lightemittingdiode; LER, luminous efficacy of radiation; RAGB, red/amber/green/blue.

We also compare the LE, luminous flux, CLE, circadian luminous flux, CRI and CQS of selected $\mathrm{R}_{\mathrm{B}, \mathrm{M}} \mathrm{A}_{\mathrm{B}, \mathrm{M}} \mathrm{G}_{\mathrm{B}, \mathrm{M}} \mathrm{B}$ (R640, A590, G515 and B450) and RAGB four-package white LEDs (Supplementary Figs. S10 and S11) at four different CCTs of $10000 \mathrm{~K}, 6500 \mathrm{~K}, 3500 \mathrm{~K}$ and $2000 \mathrm{~K}$ as a function of the applied current. These figures indicate that the $R_{B, M} A_{B, M} G_{B, M} B$ four-package white LEDs have at least similar variations of the LE, luminous flux, CLE, circadian luminous efficacy and circadian luminous flux with the current relative to the wide variation of the current stability levels of conventional semiconductor-type RAGB four-package white LEDs that do not contain phosphors. The figures also show that the current variations of both the CRI and CQS values of $R_{B, M} A_{B, M} G_{B, M} B$ LEDs are smaller than those of the RAGB fourpackage white LEDs because the dominant wavelength of the RAGB for each primary LEDs has large variations with the applied current. ${ }^{14}$

The amounts of circadian luminous flux and CIL are directly related to both the CCT value and the amount of applied current. As mentioned above, the portion of blue in the four-package $R_{B, M} A_{B, M} G_{B, M} B$ LED is similar to that of the blue light in any lamp using other technologies at the same color temperature. Therefore, the circadian differences between white light rich in blue (high CCT) and white light devoid of blue (low CCT) can be very large in terms of the effect on human health. It is possible to decrease the CCT of $R_{B, M} A_{B, M} G_{B, M} B$ four-package white LEDs below $2000 \mathrm{~K}$ and increase the total applied current to create circadian darkness at night while maintaining the same visual brightness and moderate color quality. Therefore, the circadian brightness as well as the visual brightness and color quality can be adjusted to adapt people to sleep/awake circumstances by controlling the CAF values of $R_{B, M} A_{B, M} G_{B, M} B$ four-package white LEDs and the applied current.

\section{CONCLUSIONS}

The scientific and technological advancements for developing good artificial lighting sources have a very long history since firelight was first invented by mankind. To date, all current reports that study the development and optimization of artificial lighting systems have focused on the visual performances and limited color qualities. The figures of merit, which are widely used in the lighting society, are the LER and the LE for vision performance as well as the CRI and CCT for color quality. However, these properties are insufficient when seeking to represent all performances required when ranking individual artificial lighting sources for indoor or outdoor lighting. Here, the possible figures of merit are proposed and explained to describe the circadian system, the advanced color quality and the vision performance. As shown in Figure 1, the CER, CLE, CAF, CQS, $R_{9}$ and circadian illuminance are added to the conventional figures of merit of lighting, in this case, the LER, LE, CRI and CCT values and the visual illuminance in order to assess the quality of commercialized artificial lighting sources along with our four-package $R_{B, M} A_{B, M} G_{B, M} B$ LED lighting source and standardize the daylight with respect to the vision performance, color quality and circadian performance. This analysis of the optical data of daylight and commercialized artificial lighting can provide newly developed artificial lighting sources with guidelines for attaining healthy, efficient, natural and tunable white LEDs by comparing the optical properties of the color quality, 
vision performance and circadian performance among our four-package $R_{B, M} A_{B, M} G_{B, M} B$ white LEDs, daylight and presently commercialized light sources. An additional important characteristic for optimizing all figures of merit for good artificial lightings is the capability of individual colors to control and adjust the SPDs of lighting sources to daytime and nighttime environments. The distinct color control of $R_{B, M} A_{B, M} G_{B, M} B$ LEDs combined with a narrow InGaN blue LED and three wide-band LPDF-capped green, amber, red pc-LEDs provides the capability to create tunable figures of merit while also ensuring excellent vision performances, high color qualities and a wide range of circadian effects.

More elaborate experiments are required to realize a healthy, efficient, natural and tunable white lighting system with higher circadian, vision and color qualities in which the quantum efficiency of the InGaN blue LED chip and phosphors are enhanced, the edge wavelength of the LPDF is modified and the combination of each colored LED is properly selected in a multipackage white LED system. Also, more research is needed to understand the absorption and reflection effect of the lighting in the case of indoor environments.

This study, which defines all figures of merits for good healthy lighting systems, which compares the optical properties of daylight and commercialized artificial lamps and which utilizes four-package $\mathrm{R}_{\mathrm{B}, \mathrm{M}} \mathrm{A}_{\mathrm{B}, \mathrm{M}} \mathrm{G}_{\mathrm{B}, \mathrm{M}} \mathrm{B}$ white LED systems with LPDF-capped pc-LEDs, can lead to the creation of high-quality smart lighting systems for human health, energy savings and the realization of natural colors.

\section{AUTHOR CONTRIBUTIONS}

YRD conceived and designed the experiments. JHO and SJY performed the experiments and the data analysis.

\section{ACKNOWLEDGMENTS}

This work was supported by the National Research Foundation of Korea(NRF) grant funded by the Korea government(MSIP) (No. 2011-0017449).

1 Crawford $\mathrm{MH}$. LEDs for solid-state lighting: performance challenges and recent advances. IEEE J Sel Top Quantum Electron 2009; 15: 1028-1040.

2 Schubert EF, Kim JK. Solid-state lighting sources getting smart. Science 2005; 308 : 1274-1278.

3 Pauley SM. Lighting for the human circadian clock: recent research indicates that lighting has become a public health issue. Med Hypotheses 2004; 63: 588-596.

4 Cajochen C, Münch M, Kobialka S, Kräuchi K, Steiner R et al. High sensitivity of human melatonin, alertness, thermoregulation, and hear rate to short wavelength light. J Clin Endocrinol Metab 2005; 90: 1311-1316.

5 Anderson JL, Glod CA, Dai J, Lockley SW. Lux vs. wavelength in light treatment of seasonal affective disorder. Acta Psychiatry Scand 2009; 120: 203-212.

6 Partonen T. Effects of morning light treatment on subjective sleepiness and mood in winter depression. J Affect Disord 1994; 30: 47-56.

7 Eastman Cl, Young MA, Fogg LF. Bright light treatment of winter depression: a placebo controlled trial. Arch Cen Physhiatry 1998; 55: 883-889.

8 Gooley JJ. Treatment of circadian rhythm sleep disorders with light. Ann Acad Med 2008; 37: 669-676.

9 Dowling GA, Mastick J, Colling E, Cater JH, Singer CM et al. Melatonin for sleep disturbances in Parkinson's disease. Sleep Med 2005; 6: 459-466.

10 Eastman Cl, Boulos Z, Terman M, Campbell SS, Dijk DJ et al. Light treatment for sleep disorders: consensus report. VI. Shift work. J Biol Rhythms 1995; 10: 157-164.
11 Boivin DB, Duffy JF, Kronauer RE, Czeisler CA. Dose-response relationships for restting of human circadian color by light. Nature 1996; 379: 540-542.

12 Falchi F, Cinzano P, Elvidge CD, Keith DM, Haim AL. Limiting the impact of light pollution on human health, environment and stellar visibility. J Environ Manage 2011; 92: 2714-2722.

13 Bellia L, Bisegna F, Spada G. Lighting in indoor environments: visual and non-visual effects of light sources with different spectral power distributions. Build Environ 2011; 46: 1984-1992.

14 Oh JH, Oh JR, Park HK, Sung YG, Do YR. New paradigm of multi-chip white LEDs: combination of an InGaN blue LED and full down-converted phosphor-converted LEDs. Opt Express 2011; 19: A270-A279.

15 Oh JH, Oh JR, Park HK, Sung YG, Do YR. Highly-efficient, tunable green, phosphorconverted LEDs using a long-pass dichroic filter and a series of orthosilicate phosphors for tri-color white LEDs. Opt Express 2012; 20: A1-A12.

16 Oh JH, Yang SJ, Sung YG, Do YR. Excellent color rendering indexes of multi-package white LEDs. Opt Express 2012; 20: 20276-20285.

17 Žukauskas A, Vaicekauskas R, Vitta P. Optimization of solid-state lamps for photobiologically friendly mesopic lighting. App/ Opt 2012; 51: 8423-8432.

18 Gall D, Bieske K. Definition and measurement of circadian radiometric quantities. In: Proceedings of the CIE Symposium 2004 on Light and Health: Non-Visual Effects, 30 September-2 October 2004; Vienna, Austria 2004. CIE: Wien, 2004, pp129-132.

19 Brainard GC, Hanifin JP, Greeson JM, Byrne B, Glickman G et al. Action spectrum for melatonin regulation in humans: evidence for a novel circadian photoreceptor. J Neurosci 2001; 21: 6405-6412.

20 Thapan K, Arendt J, Skene DJ. An action spectrum for melatonin suppression: evidence for a novel non-rod, non-cone photoreceptor system in humans. J Physiol 2001; 535: 261-267.

21 Rea MS, Figuerio MG, Bullough JD. Circadian photobiology: an emerging framework for lighting practice and research. Lighting Res Technol 2002; 34: 177-187.

22 Lang D. Energy efficient illumination for the biological clock. Proc SPIE 2011; 7954 795402.

23 Rea M, Figueiro MG, Bierman A, Bullough JD. Circadian light. J Circadian Rhythms 2010; 8: 2.

24 Rea MS, Figueiro MG, Bierman A, Hammer R. Modelling the spectral sensitivity of the human circadian system. Lighting Res Technol 2012; 44: 386-396.

25 Bellia L, Seraceni M. A proposal of a simplified model to evaluate circadian effects of light sources. Lighting Res Technol; e-pub ahead of print 13 June 2013; doi:10.1177/1477153513490715.

26 Aubé M, Roby J, Kocifaj M. Evaluating potential spectral impacts of various artificial lights on melatonin suppression, photosynthesis, and star visibility. PLoS ONE2013; 8: e67798.

27 Ohno Y. Spectral design considerations for white LED color rendering. Opt Eng 2005; 44: 111302.

28 Žukauskas A, Vaicekauskas R, Ivanauskas F, Vaitkevicius H, Vitta P et al. Statistical approach to color quality of solid-state lamps. IEEE J Sel Top Quantum Electron 2009; 15: 1753-1762.

29 Ohno Y. Color rendering and luminous efficacy of white LED spectra. Proc SPIE 2004; 5530: 88-98.

30 Žukauskas A, Vaicekauskas R, Shur MS. Colour-rendition properties of solid-state lamps. J phys D Appl Phys 2010; 43: 354006.

31 Davis W, Ohno Y. Toward and improved color rendering metric. Proc SPIE 2005; 5941: 59411G.

32 Davis W, Ohno Y. Color quality scale. Opt Eng 2010; 49: 033602.

33 Smet KAG, Ryckaert WR, Pointer MR, Deconinck G, Hanselar P. A memory colour quality metric for white light sources. Energy Buildings 2012; 49: 216-225.

34 Oh JR, Cho SH, Park HK, Oh JH, Lee YH et al. Full down-conversion of amber-emitting phosphor-converted light-emitting diodes with powder phosphors and a long-wave pass filter. Opt Express 2010; 18: 11063-11072.

$35 \mathrm{Oh} \mathrm{JR}$, Cho SH, Oh JH, Kim YK, Lee YH et al. The realization of a whole palette of colors in a green gap by monochromatic phosphor-converted light-emitting diodes. Opt Express 2011; 19: 4188-4198.

36 Phillips JM, Coltrin ME, Crawford MH, Fischer AJ, Krames MR et al. Research challenges to ultra-efficient inorganic solid-state lighting. Laser Photon Rev 2007; 1: 307-333.

37 Humphreys CJ. Solid-state lighting. MRS Bull 2008; 33: 459-470.

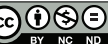

This work is licensed under a Creative Commons AttributionNonCommercial-NoDerivs Works 3.0 Unported license. To view a copy of this license, visit http://creativecommons.org/licenses/by-nc-nd/3.0 